\title{
Analytical solutions to the 3-D compressible Navier-Stokes equations with free boundaries
}

Mei Wang ${ }^{1 *}$, Zilai $\mathrm{Li}^{2}$ and Wei $\mathrm{Li}^{1}$

\author{
"Correspondence: \\ wangmei0439@163.com \\ 'School of Mathematics, Northwest \\ University, Xi'an, 710127, China \\ Full list of author information is \\ available at the end of the article
}

\begin{abstract}
In this paper, we study a class of analytical solutions to the 3-D compressible Navier-Stokes equations with density-dependent viscosity coefficients, where the shear viscosity $h(\rho)=\mu \geq 0$, and the bulk viscosity $g(\rho)=\rho^{\beta}(\beta>0)$. By constructing a class of radial symmetric and self-similar analytical solutions in $\mathbb{R}^{N}(N \geq 2)$ with both the continuous density condition and the stress free condition across the free boundaries separating the fluid from vacuum, we derive that the free boundary expands outward in the radial direction at an algebraic rate in time and we also have shown that such solutions exhibit interesting new information such as the formation of a vacuum at the center of the symmetry as time tends to infinity and explicit regularities, and we have large time decay estimates of the velocity field.
\end{abstract}

Keywords: analytical solution; compressible Navier-Stokes equations; density-dependent

\section{Introduction}

The compressible Navier-Stokes equations with density-dependent viscosity coefficients can be written as

$$
\left\{\begin{array}{l}
\partial_{t} \rho+\operatorname{div}(\rho \mathbf{U})=0 \\
\partial_{t}(\rho \mathbf{U})+\operatorname{div}(\rho \mathbf{U} \otimes \mathbf{U})-\operatorname{div}(h(\rho) D(\mathbf{U}))-\nabla(g(\rho) \operatorname{div} \mathbf{U})+\nabla P(\rho)=0,
\end{array}\right.
$$

where $\rho(\mathbf{x}, t), \mathbf{U}(\mathbf{x}, t)$, and $P(\rho)=\rho^{\gamma}(\gamma>1)$ stand for the fluid density, velocity, and pressure, respectively. The strain tensor is

$$
D(\mathbf{U})=\frac{\nabla \mathbf{U}+\nabla \mathbf{U}^{t}}{2}
$$

and $h(\rho)$ and $g(\rho)$ are the Lamé viscosity coefficients satisfying

$$
h(\rho)>0, \quad h(\rho)+N g(\rho) \geq 0 .
$$

During the last several decades, there has been significant progress on the system (1.1) since it was introduced by Liu et al. in [1]. There is a huge literature on the global exis-

(c) 2015 Wang et al. This article is distributed under the terms of the Creative Commons Attribution 4.0 International License (http://creativecommons.org/licenses/by/4.0/), which permits unrestricted use, distribution, and reproduction in any medium, provided you give appropriate credit to the original author(s) and the source, provide a link to the Creative Commons license, and indicate if changes were made. 
tence and behaviors of solutions to (1.1) in the case that the viscosity coefficients are both constants. The important progress on the global existence of strong or weak solutions in one spatial dimension or multi-dimension has been made by many authors; refer to [2-8] and references therein.

However, the studies in Hoff and Serre [9], Xin [10], and Liu et al. [1] prove that the compressible Navier-Stokes equations with constant viscosity coefficients behave singularly in the presence of a vacuum. In [1] Liu, Xin and Yang introduced the modified compressible Navier-Stokes equations with density-dependent viscosity coefficients for isentropic fluids. In deriving by Chapman-Enskog expansions from the Boltzmann equation, the viscosity of the compressible Navier-Stokes equations depends on the temperature and thus on the density for isentropic flows. Moreover, it should be emphasized that the viscous Saint-Venant system in the description of the motion for shallow water was also derived recently [11, 12], which is expressed exactly as (1.1) with $N=2, h(\rho)=\rho, g(\rho)=0$, and $\gamma=2$.

The system (1.1) was first proposed and studied by Vaigant-Kazhikhov in [13]. There is much literature on mathematical studies of the Navier-Stokes equations with densitydependent viscosities. For the one-dimensional case one is referred to [1, 14-24] and references therein. For the periodic problem on the torus $\mathbb{T}^{2}$ and under assumptions that the initial density is uniform away from vacuum and $\beta>3$ where $h(\rho)$ is a positive constant and $g(\rho)=\rho^{\beta}$, Vaigant-Kazhikhov found the well-posedness of the classical solution to (1.1), and the global existence and large time behavior of weak solutions was studied by Perepelitsa in [25]. Later on, Jiu-Wang-Xin [26] improved the result and obtained the global well-posedness of the classical solution to the periodic problem with large initial data permitting vacuum. Then Huang-Li [27] relaxed the index $\beta$ to $\beta>\frac{4}{3}$ and studied the large time behavior of the solutions. For a 2-D Cauchy problem with vacuum states at far fields, Jiu-Wang-Xin [28] and Huang-Li [29] independently considered the global well-posedness of classical solutions in different weighted spaces. Later on, Jiu-Wang-Xin [30] studied the global well-posedness of the classical solution to the Cauchy problem (1.1) with $h(\rho)$ being a positive constant and $g(\rho)=\rho^{\beta}$ and large data which keeps non-vacuum states $\bar{\rho}>0$ at far fields.

The first successful example is due to Guo et al. in [31] with spherically symmetric initial data and fixed boundary conditions, and later Guo et al. extended it to the free boundary conditions with discontinuously symmetric initial data [32]. Chen and Zhang in [33] showed a local existence result with spherically symmetric initial data between a solid core and a free boundary connected to a surrounding vacuum state.

In [32], Guo had discussed the global existence, the Lagrangian structure and large time behavior of weak solutions to the compressible spherically symmetric Navier-Stokes equations (1.1) with the following stress free boundary conditions:

$$
\rho^{\gamma}=\rho u_{r}, \quad \text { on } r=a(t),
$$

with $h(\rho)=\rho, g(\rho)=0$, and $\gamma \in\left[2, \frac{N}{N-2}\right)$. Notice that the results in [32] can also be generalized to multi-dimensional compressible spherically symmetric Navier-Stokes equation (1.1) with general viscosity coefficients $h(\rho)=\rho^{\theta}$ and $g(\rho)=(\theta-1) \rho^{\theta}$ with $\frac{N-1}{N}<\theta \leq 1$ with 
$N \geq 2$ the space dimension or supplying the following free boundary:

$$
\rho^{\gamma}=\theta \rho^{\theta}\left(u_{r}+\frac{2 u}{r}\right), \quad \text { on } r=a(t) .
$$

On the other hand, there are many references considering the analytical solutions or blowup solutions to the Navier-Stokes equations, Navier-Stokes-Poisson equations, or Euler-Poisson equations (see [34-36]). In [37], Guo has studied the analytical solutions to the compressible Navier-Stokes equations with density-dependent viscosity coefficients where $h(\rho)=\rho^{\theta}$ and $g(\rho)=(\theta-1) \rho^{\theta}, N=3$, and with free boundaries.

In the present paper, we study more precisely the structure and asymptotic behavior of solutions to the free boundary value problem with both the continuous density condition and the stress free condition. Let $h(\rho)=\mu \geq 0$ and $g(\rho)=\rho^{\beta}(\beta>0)$. At first, we construct a self-similar solution of the form

$$
\rho(r, t)=\frac{f\left(\frac{r}{a(t)}\right)}{a^{3}(t)}, \quad u(r, t)=\frac{a^{\prime}(t)}{a(t)} r,
$$

to the continuity equation with special spherically symmetric initial data and given symmetric sphere separating the initial fluids from vacuum, and we derive that $a(t), f(z) \in C^{1}$ are two functions satisfying some ordinary differential equations. At the same time, the free boundary is shown to expand outward in the radial direction at an algebraic rate in time and the density decays to zero asymptotically in time almost everywhere away from the symmetry center, which leads to the formation of vacuum states as the time goes to infinity. One of our main steps in the analysis is to show that $a(t)$ and $f(z) \in C^{1}$ exist globally, which yields the desired analytical solutions to the free boundary value problem with suitable initial data.

The plan of this paper is as follows. In Section 2 we will give the main results and the self-similar solution to (1.1). Theorems 2.1 and 2.2 will be proved in Sections 3.1 and 3.2, respectively.

\section{Notations and main results}

Set $h(\rho)=\mu \geq 0$ and $g(\rho)=\rho^{\beta}, N=3$, in (1.1). Then the isentropic compressible NavierStokes system with density-dependent viscosity becomes

$$
\left\{\begin{array}{l}
\partial_{t} \rho+\operatorname{div}(\rho \mathbf{U})=0, \\
\partial_{t}(\rho \mathbf{U})+\operatorname{div}(\rho \mathbf{U} \otimes \mathbf{U})+\nabla P(\rho)=\mu \Delta \mathbf{U}+\nabla\left(\left(\mu+\rho^{\beta}\right) \operatorname{div}(\mathbf{U})\right),
\end{array}\right.
$$

for $t \in(0,+\infty)$ and $\mathbf{x} \in \mathbb{R}^{3}$. Here $\rho(\mathbf{x}, t), \mathbf{U}(\mathbf{x}, t)$, and $P(\rho)=\rho^{\gamma}\left(\gamma>\frac{4}{3}\right)$. The initial conditions of (2.1) are imposed thus:

$$
\left.(\rho, \rho \mathbf{U})\right|_{t=0}(\mathbf{x})=\left(\rho_{0}, \mathbf{m}_{0}\right)(\mathbf{x}), \quad 0 \leq|\mathbf{x}| \leq a_{0} .
$$

In this paper, we are concerned with the following spherically symmetric solutions. To this end, we denote

$$
|\mathbf{x}|=r, \quad \rho(\mathbf{x}, t)=\rho(r, t), \quad \mathbf{U}(\mathbf{x}, t)=u(r, t) \frac{\mathbf{x}}{r} .
$$


This leads to the following system of equations:

$$
\begin{aligned}
& \rho_{t}+(\rho u)_{r}+\frac{2 \rho u}{r}=0, \\
& (\rho u)_{t}+\left(\rho u^{2}+\rho^{\gamma}\right)_{r}+\frac{2 \rho u^{2}}{r}=\left(\left(2 \mu+\rho^{\beta}\right)\left(u_{r}+\frac{2 u}{r}\right)\right)_{r},
\end{aligned}
$$

for $(r, t) \in \Omega$ with

$$
\Omega=\{(r, t) \mid 0 \leq r \leq a(t), t \geq 0\}
$$

with the initial condition

$$
\left.(\rho, u)\right|_{t=0}=\left(\rho_{0}(r), u_{0}(r)\right), \quad 0 \leq r \leq a_{0},
$$

where $a_{0}>0$ is a constant, and we have the free boundary condition

$$
\rho(a(t), t)=0,
$$

or, when $\mu=0$,

$$
\rho^{\gamma}=\rho^{\beta}\left(u_{r}+\frac{2 u}{r}\right), \quad \text { on } a(t)
$$

where

$$
a^{\prime}(t)=u(a(t), t), \quad a(0)=a_{0}, \quad \forall t \geq 0 .
$$

It is easy to get the following usual a priori energy estimate for smooth solutions to (2.4), (2.5), and the boundary conditions (2.7) or (2.8):

$$
\frac{d}{d t} \int_{0}^{a(t)}\left(\frac{1}{2} \rho u^{2}+\frac{1}{\gamma-1} \rho^{\gamma}\right) r^{2} d r+\int_{0}^{a(t)}\left(2 \mu+\rho^{\beta}\right)\left(u_{r} r+2 u\right)^{2} d r \leq 0 .
$$

Our main result is the following theorem.

Theorem 2.1 Assume that $\gamma=\beta>\frac{4}{3}$. For the radial symmetry compressible Navier-Stokes equations (2.4)-(2.5), with the continuous density condition (2.7), there exists a solution of the form

$$
\rho(r, t)=\frac{\left[\frac{\gamma-1}{2 \gamma}\left(1-\frac{r^{2}}{a^{2}(t)}\right)\right]^{\frac{1}{\gamma-1}}}{a^{3}(t)}, \quad u(r, t)=\frac{a^{\prime}(t)}{a(t)} r .
$$

Here $a(t) \in C^{1}([0, \infty))$ is the free boundary satisfying (2.9) and it exists for all $t \geq 0$. Furthermore, $a(t)$ tends to $+\infty$ as $t \rightarrow+\infty$ with the following rates:

$$
C_{1}(1+t)^{\frac{1}{3(\gamma-1)}} \leq a(t) \leq C_{2}(1+t)
$$


Remark 2.1 The solution (2.11) constructed in Theorem 2.1 satisfies the following properties:

$$
\begin{aligned}
& \rho(0, t) \rightarrow 0, \quad \text { as } t \rightarrow+\infty, \\
& |\Omega(t)|=\frac{4}{3} \pi a^{3}(t) \rightarrow+\infty, \quad \text { as } t \rightarrow+\infty,
\end{aligned}
$$

where the domain of the fluid is defined by

$$
\Omega(t)=\{(r, t) \mid 0 \leq r \leq a(t), t \geq 0\} .
$$

On the other hand, for the stress free boundary condition (2.8), we have the following.

Theorem 2.2 Assume that $\gamma=2 \beta-\frac{1}{3}, \gamma>\frac{5}{3}$. Then the free boundary value problem for the radial symmetry compressible Navier-Stokes system, (2.4)-(2.5), with the stress free condition (2.8), has a unique solution with the free boundary $r=a(t)$ given by

$$
a(t)=f^{\frac{1}{3}}(1)\left(\rho_{0}^{\beta-\gamma}\left(a_{0}\right)+(\gamma-\beta) t\right)^{\frac{1}{3(\gamma-\beta)}}
$$

and

$$
\rho(r, t)=\frac{f\left(\frac{r}{a(t)}\right)}{a^{3}(t)}, \quad u(r, t)=\frac{r}{3\left(\rho_{0}^{\beta-\gamma}\left(a_{0}\right)+(\gamma-\beta) t\right)},
$$

where $f(z)>0, f(z) \in C([0,1]) \cap C^{1}((0,1])$ satisfies

$$
\frac{1-\gamma}{2} z+\gamma f(1)^{\frac{1}{3}-\gamma} f^{\gamma-2}(z) f^{\prime}(z)-\frac{1}{2}\left(\gamma+\frac{1}{3}\right) f(1)^{\frac{1}{6}-\frac{\gamma}{2}} f^{\frac{3 \gamma-11}{6}}(z) f^{\prime}(z)=0 .
$$

Remark 2.2 It can be verified easily that the solution (2.15) in Theorem 2.2, satisfies the following properties:

$$
\begin{aligned}
& \rho(0, t), \rho(a(t), t) \rightarrow 0, \quad \text { as } t \rightarrow+\infty, \\
& |\Omega(t)|=\frac{4}{3} \pi a^{3}(t) \rightarrow+\infty, \quad \text { as } t \rightarrow+\infty .
\end{aligned}
$$

Moreover, for $0 \leq r \leq a(t)$, we have

$$
\begin{aligned}
& |u(r, t)|=\frac{\left|a^{\prime}(t)\right|}{a(t)} r \leq\left|a^{\prime}(t)\right| \leq C(1+t)^{\frac{1-\gamma}{\gamma-\frac{1}{3}}} \rightarrow 0, \quad \text { as } t \rightarrow+\infty, \\
& \left|u_{r}(r, t)\right|=\frac{1}{3\left(\rho_{0}^{\beta-\gamma}\left(a_{0}\right)+(\gamma-\beta) t\right)} \rightarrow 0, \quad \text { as } t \rightarrow+\infty .
\end{aligned}
$$

\section{The proofs of the main theorems}

First, we will give a self-similar solution to the continuity equation (2.4).

Lemma 3.1 For any two $C^{1}$ functions $f(z) \geq 0$ and $a(t)>0$, define

$$
\rho(r, t)=\frac{f\left(\frac{r}{a(t)}\right)}{a^{3}(t)}, \quad u(r, t)=\frac{a^{\prime}(t)}{a(t)} r .
$$


Then $(\rho, u)(r, t)$ solves the continuity equation (2.4), i.e.,

$$
\rho_{t}+(\rho)_{r} u+\rho u_{r}+\frac{2 \rho u}{r}=0 .
$$

Here, we can choose $a(t)$ as a free boundary which satisfies the condition (2.7) or (2.8). So in the following we will determine the form of the function $f(x)$ and then prove the global existence of the free boundary $a(t)$. To this end, denoting $z=\frac{r}{a(t)}$, one can obtain from (2.5) that

$$
\frac{a^{\prime \prime}(t)}{a^{3}(t)} f(z) z+\gamma f^{\gamma-1}(z) f^{\prime}(z) \frac{1}{a^{3 \gamma+1}(t)}-\frac{3 a^{\prime}(t)}{a^{3 \beta+2}(t)} \beta f^{\beta-1}(z) f^{\prime}(z)=0 .
$$

Next, we will solve (3.3) according to the free boundary conditions (2.7) or (2.8), respectively.

\subsection{The continuous boundary condition}

Assume that $\gamma=\beta>\frac{4}{3}$. We require

$$
z=-\gamma f^{\gamma-2}(z) f^{\prime}(z)
$$

Then it follows from (3.3)-(3.4) that

$$
\begin{aligned}
& f(z)=\left[f^{\gamma-1}(1)+\frac{\gamma-1}{2 \gamma}\left(1-z^{2}\right)\right]^{\frac{1}{\gamma-1}}, \\
& a^{\prime \prime}(t)-a^{2-3 \beta}(t)+3 a^{\prime}(t) a^{1-3 \beta}(t)=0 .
\end{aligned}
$$

Using the boundary condition (2.7) yields $f(z)=\left[\frac{\gamma-1}{2 \gamma}\left(1-z^{2}\right)\right]^{\frac{1}{\gamma-1}}$, and then

$$
\rho(r, t)=\frac{\left[\frac{\gamma-1}{2 \gamma}\left(1-z^{2}\right)\right]^{\frac{1}{\gamma-1}}}{a^{3}(t)}, \quad u(r, t)=\frac{a^{\prime}(t)}{a(t)} r .
$$

Clearly, if $a(t)>0$ is a free boundary satisfying the condition (2.7), then it is straightforward to check that $(\rho, u)$ defined by (3.7) is a solution of (2.4)-(2.5), where $a(t)$ can be determined by

$$
\left\{\begin{array}{l}
a^{\prime}(t)=a_{1}+\int_{0}^{t} a^{2-3 \beta}(s) d s-\frac{3}{2-3 \beta} a^{2-3 \beta}(t)+\frac{3}{2-3 \beta} a_{0}^{2-3 \beta}, \\
a(0)=a_{0}, \quad a^{\prime}(0)=a_{1},
\end{array}\right.
$$

with $a_{0}>0$ and $a_{1}$ being the initial location and slope of the free boundary.

Thus, it remains to solve the boundary value problem (3.8). We start with estimates on solutions of (3.8).

Lemma 3.2 Let $a(t) \in C^{1}[0, T]$ be a solution to (3.8) for $T>0$. Then there exist two uniform positive constants $C_{1}$ and $C_{2}>0$, independent of $T$, such that

$$
C_{1}(1+t)^{\frac{1}{3(\gamma-1)}} \leq a(t) \leq C_{2}(1+t)^{\frac{6 \beta-4}{3(\beta-1)}} .
$$


Proof We first verify the fact that $a(t) \geq 1$ for all $t \in[0, T]$. Note that (3.7) implies

$$
\rho(a(t), t)=0, \quad u(0, t)=0 .
$$

We introduce

$$
\begin{aligned}
H(t)= & \int_{0}^{a(t)}(r-(1+t) u)^{2} \rho r^{2} d r+\frac{2}{\gamma-1}(1+t)^{2} \int_{0}^{a(t)} \rho^{\gamma} r^{2} d r \\
= & \int_{0}^{a(t)} \rho r^{4} d r-2(1+t) \int_{0}^{a(t)} \rho u r^{3} d r+(1+t)^{2} \int_{0}^{a(t)} \rho u^{2} r^{2} d r \\
& +\frac{2}{\gamma-1}(1+t)^{2} \int_{0}^{a(t)} \rho^{\gamma} r^{2} d r .
\end{aligned}
$$

Due to $a^{\prime}(t)=u(a(t), t)$ and (3.10), a direct computation gives

$$
\begin{aligned}
H^{\prime}(t)= & \int_{0}^{a(t)}\left(\rho_{t} r^{4}-2 \rho u r^{3}\right) d r+(1+t)^{2} \int_{0}^{a(t)}\left(\left(\rho u^{2}\right)_{t}+\frac{2}{\gamma-1}\left(\rho^{\gamma}\right)_{t}\right) r^{2} d r \\
& +2(1+t) \int_{0}^{a(t)}\left(\rho u^{2} r^{2}-(\rho u)_{t} r^{3}+\frac{2}{\gamma-1} \rho^{\gamma} r^{2}\right) d r \\
= & I_{1}+I_{2}+I_{3} .
\end{aligned}
$$

Equations (2.4) and (3.10) yield

$$
I_{1}=-\int_{0}^{a(t)}\left(\rho u r^{4}\right)_{r} d r=0 .
$$

One has

$$
\begin{aligned}
I_{2}= & (1+t)^{2} \int_{0}^{a(t)}\left(\left(\rho u^{2}\right)_{t}+\frac{2}{\gamma-1}\left(\rho^{\gamma}\right)_{t}\right) r^{2} d r \\
= & (1+t)^{2} \int_{0}^{a(t)} 2(\rho u)_{t} u r^{2}-\rho_{t} u^{2} r^{2}+\frac{2 \gamma}{\gamma-1} \rho^{\gamma-1} \rho_{t} r^{2} d r \\
= & (1+t)^{2} \int_{0}^{a(t)} 2 u\left[-\left(\rho u^{2}\right)_{r} r^{2}-\left(\rho^{\gamma}\right)_{r} r^{2}\right]-\frac{2 \gamma}{\gamma-1} \rho^{\gamma-1}(\rho u)_{r} r^{2} \\
& -\frac{2 \gamma}{\gamma-1} \rho^{\gamma} u 2 \gamma+(\rho u)_{r} u^{2} r^{2}-2 \rho u^{3} r+2 u r^{2}\left(\left(2 \mu+\rho^{\gamma}\right)\left(u_{r}+\frac{2 u}{r}\right)\right)_{r} d r \\
= & (1+t)^{2} \int_{0}^{a(t)}-\frac{2 \gamma}{\gamma-1}\left(\rho^{\gamma} u r^{2}\right)_{r}-\left(\rho u^{3} r^{2}\right)_{r}+2 u r^{2}\left(\left(2 \mu+\rho^{\gamma}\right)\left(u_{r}+\frac{2 u}{r}\right)\right)_{r} d r \\
= & (1+t)^{2} \int_{0}^{a(t)} 2 u r^{2}\left((2 \mu+\lambda(\rho))\left(u_{r}+\frac{2 u}{r}\right)\right)_{r} d r \\
= & (1+t)^{2} \int_{0}^{a(t)}\left[\left(\rho^{\gamma}\right)_{r}\left(2 u u_{r} r^{2}+4 u^{2} r\right)+\left(2 \mu+\rho^{\gamma}\right)\left(4 u u_{r} r-4 u^{2}\right)\right] \\
= & -(1+t)^{2} \int_{0}^{a(t)} \rho^{\gamma}\left(2 u u_{r} r^{2}+4 u^{2} r\right)_{r}+(1+t)^{2} \int_{0}^{a(t)}\left(2 \mu+\rho^{\gamma}\right)\left(4 u u_{r} r-4 u^{2}\right) \\
= & -(1+t)^{2} \int_{0}^{a(t)}\left(2 \rho^{\gamma} u_{r}^{2} r^{2}+16 \rho^{\gamma} u^{2}\right) d r,
\end{aligned}
$$




$$
\begin{aligned}
I_{3}= & 2(1+t) \int_{0}^{a(t)} \rho u^{2} r^{2}-(\rho u)_{t} r^{3}+\frac{2}{\gamma-1} \rho^{\gamma} r^{2} d r \\
= & 2(1+t) \int_{0}^{a(t)} \frac{5-3 \gamma}{\gamma-1} \rho^{\gamma} r^{2}-\left(\left(2 \mu+\rho^{\gamma}\right)\left(u_{r}+\frac{2 u}{r}\right)\right)_{r} r^{3} d r \\
= & 2(1+t) \int_{0}^{a(t)} \rho^{\gamma}\left(u_{r} r^{3}+2 u r^{2}\right)_{r} \\
& -2(1+t) \int_{0}^{a(t)}\left(2 \mu+\rho^{\gamma}\right)\left(2 u_{r} r^{2}-2 u r\right)+\frac{5-3 \gamma}{\gamma-1} \rho^{\gamma} r^{2} d r \\
= & 2(1+t) \int_{0}^{a(t)} 3 \rho^{\gamma} u_{r} r^{2}+8 \rho^{\gamma} u r+\frac{5-3 \gamma}{\gamma-1} \rho^{\gamma} r^{2} d r .
\end{aligned}
$$

Thus, substituting the above estimates into (3.12) and using the Cauchy-Schwarz inequality, one deduces

$$
\begin{aligned}
H^{\prime}(t)= & -(1+t)^{2} \int_{0}^{a(t)}\left(2 \rho^{\gamma} u_{r}^{2} r^{2}+16 \rho^{\gamma} u^{2}\right) d r+(1+t) \int_{0}^{a(t)} 6 \rho^{\gamma} u_{r} r^{2} \\
& +16 \rho^{\gamma} u r+\frac{5-3 \gamma}{\gamma-1} \rho^{\gamma} r^{2} d r \\
\leq & \frac{17}{2} \int_{0}^{a(t)} \rho^{\gamma} r^{2} d r+\frac{2(5-3 \gamma)}{\gamma-1}(1+t) \int_{0}^{a(t)} \rho^{\gamma} r^{2} d r
\end{aligned}
$$

where one has used

$$
\begin{aligned}
& 2(1+t) \int_{0}^{a(t)} 3 \rho^{\gamma} u_{r} r^{2} d r \leq 2(1+t)^{2} \int_{0}^{a(t)} \rho^{\gamma} u_{r}^{2} r^{2} d r+\frac{9}{2} \int_{0}^{a(t)} \rho^{\gamma} r^{2} d r, \\
& 16(1+t) \int_{0}^{a(t)} \rho^{\gamma} u r d r \leq 16(1+t)^{2} \int_{0}^{a(t)} \rho^{\gamma} u^{2} d r+4 \int_{0}^{a(t)} \rho^{\gamma} r^{2} d r .
\end{aligned}
$$

Note also that the conservation of total mass implies that

$$
\int_{0}^{a(t)} \rho r^{2} d r=\int_{0}^{a_{0}} \rho_{0} r^{2} d r=1
$$

In the case $\gamma \geq \frac{5}{3}$, (3.13) yields

$$
H^{\prime}(t) \leq \frac{17(\gamma-1)}{2} E_{0}, \quad E_{0}=\int_{0}^{a_{0}}\left(\frac{1}{2} \rho_{0} u_{0}^{2}+\frac{\rho_{0}^{\gamma}}{\gamma-1}\right) r^{2} d r
$$

and so

$$
H(t) \leq H(0)+\frac{17(\gamma-1)}{2} E_{0} t,
$$

and consequently

$$
\int_{0}^{a(t)} \rho^{\gamma} r^{2} d r \leq C(1+t)^{-1}
$$


Thus, as a consequence of (3.16) and the conservation of mass, we have, for any $t>0$,

$$
1=\int_{0}^{a(t)} \rho r^{2} d r \leq\left(\int_{0}^{a(t)} \rho^{\gamma} r^{2} d r\right)^{\frac{1}{\gamma}}\left(\int_{0}^{a(t)} r^{2} d r\right)^{\frac{\gamma-1}{\gamma}} \leq C a(t)^{3-\frac{3}{\gamma}}(1+t)^{-\frac{1}{\gamma}},
$$

which implies

$$
a(t) \geq C(1+t)^{\frac{1}{3(\gamma-1)}} .
$$

For $1<\gamma<\frac{5}{3}$, (3.13) yields

$$
\left(H(t)(1+t)^{3 \gamma-5}\right)^{\prime} \leq C(1+t)^{3 \gamma-5}
$$

which yields

$$
H(t) \leq C(1+t)^{\alpha}
$$

Here

$$
\alpha= \begin{cases}1 & \text { if } \frac{4}{3}<\gamma<\frac{5}{3}, \\ 1+\sigma, \quad \sigma>0 \text { small, } & \text { if } \gamma=\frac{4}{3}, \\ 5-3 \gamma, & \text { if } 1<\gamma<\frac{4}{3} .\end{cases}
$$

As in (3.15)-(3.17), one can show that

$$
a(t) \geq C(1+t)^{\frac{2-\alpha}{3(\gamma-1)}}
$$

Next, we derive an upper bound for $a(t)$. It follows from (3.8), (3.20) that

$$
\begin{aligned}
a^{\prime}(t) & \leq\left|a_{1}\right|+C_{1}(1+t)^{\frac{3 \beta-1}{3(\beta-1)}}+C_{2}(1+t)^{\frac{(2-\alpha)(2-3 \beta)}{3(\beta-1)}}+C_{3} a_{0}^{2-3 \beta} \\
& \leq\left|a_{1}\right|+C(1+t)^{\frac{3 \beta-1}{3(\beta-1)}}+C_{2}(1+t)^{\frac{(2-3 \beta)}{3(\beta-1)}}+C_{3} a_{0}^{2-3 \beta} .
\end{aligned}
$$

Then

$$
a(t) \leq C t+C(1+t)^{\frac{6 \beta-4}{3(\beta-1)}} \leq C(1+t)^{\frac{6 \beta-4}{3(\beta-1)}} .
$$

This yields (3.9) and completes the proof of Lemma 3.2.

We are now ready to give the existence and uniqueness of the solution to the boundary value problem (3.8).

Lemma 3.3 There exists a sufficiently small T such that (3.8) has a solution a(t), which is unique in $C^{1}[0, T]$ and satisfied with $0<\frac{1}{2} a_{0}<a(t)<2 a_{0}$.

Proof The lemma can be proved by a fixed point argument. In fact, set

$$
g(a(t))=a_{1}+\int_{0}^{t} a^{2-3 \beta}(s) d s-\frac{3}{2-3 \beta} a^{2-3 \beta}(t)+\frac{3}{2-3 \beta} a_{0}^{2-3 \beta} .
$$


Then (3.8) can be rewritten as

$$
\frac{d a(t)}{d t}=g(a(t)), \quad a(0)=a_{0}, \quad g(a(0))=a^{\prime}(0)=a_{1} .
$$

Let $T_{1}$ be a positive small constant to be determined. Define

$$
X=\left\{a(t) \in C^{1}\left[0, T_{1}\right], 0<\frac{1}{2} a_{0}<a(t)<2 a_{0}, \forall t \in\left[0, T_{1}\right]\right\} .
$$

Then, for any $a_{1}(t)$ and $a_{2}(t) \in X$, since $\beta>1$, we have

$$
\begin{aligned}
\left|g\left(a_{1}(t)\right)-g\left(a_{2}(t)\right)\right|= & \left|\int_{0}^{t}\left(a_{1}^{2-3 \beta}(s)-a_{2}^{2-3 \beta}(s)\right) d s+\frac{3}{2-3 \beta}\left(a_{2}^{2-3 \beta}(t)-a_{1}^{2-3 \beta}(t)\right)\right| \\
\leq & \frac{3}{2-3 \beta}\left(\frac{1}{2} a_{0}\right)^{4-6 \beta}\left|a_{1}-a_{2}\right|^{3 \beta-2}(t) \\
& +\left(\frac{1}{2} a_{0}\right)^{4-6 \beta} \int_{0}^{t}\left|a_{1}(s)-a_{2}(s)\right|^{3 \beta-2} d s \\
\leq & \left(\frac{1}{2} a_{0}\right)^{4-6 \beta}\left(\frac{3}{2-3 \beta}+T_{1}\right) \sup _{0 \leq t \leq T_{1}}\left|a_{1}(t)-a_{2}(t)\right|^{3 \beta-2} \\
\leq & L \sup _{0 \leq t \leq T_{1}}\left|a_{1}(t)-a_{2}(t)\right|,
\end{aligned}
$$

where $L=\left(\frac{1}{2} a_{0}\right)^{1-3 \beta} 3^{3(\beta-1)}\left(\frac{3}{2-3 \beta}+T_{1}\right)$ is a constant. We now define a mapping on $X$ by

$$
\mathbb{T} a(t)=a_{0}+\int_{0}^{t} g(a(s)) d s, \quad \forall t \in\left[0, T_{1}\right]
$$

Then $\mathbb{T} a(t) \in C^{1}\left[0, T_{1}\right]$, and, for any $t<T_{1}$, one can deduce that

$$
\begin{aligned}
\mathbb{T} a(t) & =a_{0}+\int_{0}^{t}\left(a_{1}+\int_{0}^{s} a^{2-3 \beta}(\tau) d \tau-\frac{3}{2-3 \beta} a^{2-3 \beta}(s)+\frac{3}{2-3 \beta} a_{0}^{2-3 \beta}\right) d s \\
& \leq a_{0}+\left(\left|a_{1}\right|-\frac{3}{2-3 \beta}\left(\frac{1}{2} a_{0}\right)^{2-3 \beta}\right) t+\left(\frac{1}{2} a_{0}\right)^{2-3 \beta} t^{2} \\
& \leq 2 a_{0}
\end{aligned}
$$

if

$$
t \leq \frac{\sqrt{\left(\left|a_{1}\right|-\frac{3}{2-3 \beta}\left(\frac{1}{2} a_{0}\right)^{2-3 \beta}\right)^{2}+4 a_{0}\left(\frac{1}{2} a_{0}\right)^{2-3 \beta}}-\left(\left|a_{1}\right|-\frac{3}{2-3 \beta}\left(\frac{1}{2} a_{0}\right)^{2-3 \beta}\right)}{2\left(\frac{1}{2} a_{0}\right)^{2-3 \beta}}=T_{2}
$$

and

$$
\mathbb{T} a(t) \geq a_{0}-\left(\left|a_{1}\right|-\frac{3}{2-3 \beta} a_{0}^{2-3 \beta}\right) t \geq \frac{1}{2} a_{0}
$$

if

$$
t \leq \frac{\frac{1}{2} a_{0}}{\left|a_{1}\right|-\frac{3}{2-3 \beta} a_{0}^{2-3 \beta}}=T_{3}
$$


Thus, if $T_{1} \leq \min \left\{T_{2}, T_{3}\right\}$, then $\mathbb{T} a(t) \in X$. Furthermore, since

$$
\left|\mathbb{T} a_{1}(s)-\mathbb{T} a_{2}(s)\right|=\left|\int_{0}^{t} g\left(a_{1}(s)\right) d s-\int_{0}^{t} g\left(a_{2}(s)\right) d s\right| \leq L T_{1} \sup _{0 \leq t \leq T_{1}}\left|a_{1}(t)-a_{2}(t)\right|,
$$

$\mathbb{T}$ will be a contraction mapping if $\left(\frac{3}{2-3 \beta}+T_{1}\right) T_{1}<\left(\frac{1}{2} a_{0}\right)^{3 \beta-1} 3^{3(1-\beta)}$, i.e.,

$$
T_{1}<\frac{\sqrt{\left(\frac{3}{2-3 \beta}\right)^{2}+4\left(\frac{1}{2} a_{0}\right)^{3 \beta-1} 3^{3(1-\beta)}}-\frac{3}{2-3 \beta}}{2}=T_{4} .
$$

The above argument shows that $\mathbb{T}: X \rightarrow X$ is a contraction with the sup-norm for any $T_{1}=\min \left\{T_{2}, T_{3}, T_{4}\right\}$. By the contraction mapping theorem, there exists a unique $a(t) \in$ $C^{1}\left[0, T_{1}\right]$ such that $\mathbb{T} a(t)=a(t)$ and then $a^{\prime}(t)=g(a(t))$, which yields (3.8). This completes the proof of the lemma.

Now, Theorem 2.1 follows from Lemma 3.3, the a priori estimates, Lemma 3.2, and the standard continuity argument. This completes the proof of Theorem 2.1.

\subsection{The stress free boundary condition}

First, it follows from the free boundary (2.8) and from (2.4) that

$$
\rho(a(t))=\left(\rho_{0}^{\beta-\gamma}\left(a_{0}\right)+(\gamma-\beta) t\right)^{\frac{1}{\beta-\gamma}}
$$

Using the ansatz in (3.1) shows that

$$
a(t)=f^{\frac{1}{3}}(1)\left(\rho_{0}^{\beta-\gamma}\left(a_{0}\right)+(\gamma-\beta) t\right)^{\frac{1}{3(\gamma-\beta)}}
$$

Then (3.1) becomes

$$
\rho(r, t)=\frac{f\left(\frac{r}{a(t)}\right)}{a^{3}(t)}, \quad u(r, t)=\frac{r}{3\left(\rho_{0}^{\beta-\gamma}\left(a_{0}\right)+(\gamma-\beta) t\right)} .
$$

Set $\alpha=f(1)$, and then (3.22) tells us that $\alpha>0$. Recall the assumption that $\beta=\frac{1}{2}\left(\gamma+\frac{1}{3}\right)$, $\gamma>\frac{5}{3}$. Then (3.3) becomes

$$
\frac{1-\gamma}{2} z+\gamma \alpha^{\frac{1}{3}-\gamma} f^{\gamma-2}(z) f^{\prime}(z)-\frac{1}{2}\left(\gamma+\frac{1}{3}\right) \alpha^{\frac{1}{6}-\frac{\gamma}{2}} f^{\frac{3 \gamma-11}{6}}(z) f^{\prime}(z)=0 .
$$

Denoting $g(z)=\left(\frac{f(z)}{\alpha}\right)^{\frac{3 \gamma-5}{6}}$ for any $z \in[0,1]$, then the above equality becomes

$$
\left\{\begin{array}{l}
g^{\prime}(z)\left\{g^{\frac{3 \gamma-1}{3 \gamma-5}}(z)-\frac{3 \gamma+1}{6 \gamma}\right\}=\frac{\alpha^{\frac{2}{3}}(\gamma-1)(3 \gamma-5) z}{12 \gamma} \\
g(1)=1
\end{array}\right.
$$

Next, we will prove that (3.25) can be solved on $[0,1]$. To this end, we start with a priori estimates and the uniqueness. 
Lemma 3.4 For any $\gamma>\frac{5}{3}$, let $g(z)$ be a solution to the system $(3.25)$ in $C([0,1]) \cap C^{1}((0,1])$. Then

$$
\left(\frac{3 \gamma+1}{6 \gamma}\right)^{\frac{3 \gamma-5}{3 \gamma-1}}<g(z) \leq 1
$$

for all $z \in(0,1]$. Furthermore, such a solution is unique.

Proof If $g^{\frac{3 \gamma-1}{3 \gamma-5}}(z)-\frac{3 \gamma+1}{6 \gamma}=0$, then (3.25) implies that $z$ must be zero, i.e. $g^{\frac{3 \gamma-1}{3 \gamma-5}}(0)=\frac{3 \gamma+1}{6 \gamma}$. Namely, if $z \neq 0$, then one has $g^{\frac{3 \gamma-1}{3 \gamma-5}}(z) \neq \frac{3 \gamma+1}{6 \gamma}$.

If for any $z \in(0,1], g^{\frac{3 \gamma-1}{3 \gamma-5}}(z) \in\left[0, \frac{3 \gamma+1}{6 \gamma}\right)$, then (3.25) implies that $g^{\prime}(z) \leq 0$ and thus

$$
1 \leq g(z)<\left(\frac{3 \gamma+1}{6 \gamma}\right)^{\frac{3 \gamma-5}{3 \gamma-1}}<1
$$

which is a contradiction. Thus we can deduce that $g^{\frac{3 \gamma-1}{3 \gamma-5}}(z)>\frac{3 \gamma+1}{6 \gamma}$ for all $z \in(0,1]$ and together with (3.25) to get $g^{\prime}(z) \geq 0$ and consequently

$$
\left(\frac{3 \gamma+1}{6 \gamma}\right)^{\frac{3 \gamma-5}{3 \gamma-1}}<g(z) \leq 1, \quad \forall z \in(0,1] .
$$

It remains to prove the uniqueness.

To this end, let $\bar{g}(z) \in C([0,1]) \cap C^{1}((0,1])$ be another solution to (3.25) with $\bar{g}(1)=1$ and $\left(\frac{3 \gamma+1}{6 \gamma}\right)^{\frac{3 \gamma-5}{3 \gamma-1}}<g(z) \leq 1$ for all $z \in(0,1]$.

Define $w(z)=g(z)-\bar{g}(z)$. Then $w(z)$ solves the following problem:

$$
\left\{\begin{array}{l}
\frac{d}{d z}\left\{w(z)-\frac{\gamma(3 \gamma-5)}{(3 \gamma+1)(\gamma-1)}\left\{[w(z)+\bar{g}(z)]^{\frac{6(\gamma-1)}{3 \gamma-5}}-\bar{g}(z)^{\frac{6(\gamma-1)}{3 \gamma-5}}\right\}\right\}=0, \\
w(1)=0, \quad \bar{g}(1)=1 .
\end{array}\right.
$$

Set

$$
I=\{z \in[0,1] \mid w(\xi) \equiv 0, z \leq \xi \leq 1\} .
$$

Here $I \neq \emptyset$ because of $1 \in I$. Define $z_{0}=\inf I$ and then $z_{0} \in[0,1]$. Obviously, the uniqueness of solutions to the system (3.25) will be shown by proving that $z_{0} \equiv 0$ and using a continuity argument.

If not, then $z_{0} \in(0,1]$, and $w\left(z_{0}\right)=0$. For any $z \in\left(0, z_{0}\right),(3.26)$ tells us that

$$
\left(\frac{3 \gamma+1}{6 \gamma}\right)^{\frac{3 \gamma-5}{3 \gamma-1}}<g(z) \leq 1, \quad \forall z \in\left(0, z_{0}\right)
$$

Integrating (3.28) over $\left[z, z_{0}\right]$ shows

$$
w(z)-\frac{\gamma(3 \gamma-5)}{(3 \gamma+1)(\gamma-1)}\left\{[w(z)+\bar{g}(z)]^{\frac{6(\gamma-1)}{3 \gamma-5}}-\bar{g}(z)^{\frac{6(\gamma-1)}{3 \gamma-5}}\right\}=0 .
$$


Since for any $\gamma>\frac{5}{3}$ and $\frac{6(\gamma-1)}{3 \gamma-5}>2$, a Taylor expansion gives

$$
[w(z)+\bar{g}(z)]^{\frac{6(\gamma-1)}{3 \gamma-5}}-(\bar{g}(z))^{\frac{6(\gamma-1)}{3 \gamma-5}}=\frac{6(\gamma-1)}{3 \gamma-5}[\bar{g}(z)]^{\frac{3 \gamma-1}{3 \gamma-5}} w(z)+O(1) w^{2}(z)
$$

for sufficiently small $w(z)$. Putting (3.31) into (3.30) and using the fact $w\left(z_{0}\right)=0$, one has

$$
\left\{1-\frac{6 \gamma}{3 \gamma+1}[\bar{g}(z)]^{\frac{3 \gamma-1}{3 \gamma-5}}\right\} w(z)-\frac{\gamma(3 \gamma-5)}{(3 \gamma+1)(\gamma-1)} O(1) w^{2}(z)=0
$$

for $z$ close to $z_{0}$. Notice that

$$
1-\frac{6 \gamma}{3 \gamma+1}[\bar{g}(z)]^{\frac{3 \gamma-1}{3 \gamma-5}}<0, \quad \forall z \in\left(0, z_{0}\right)
$$

by virtue of (3.29). Then one can easily deduce that $w(z) \equiv 0, \forall z \in\left(z_{0}-\delta, z_{0}\right)$ for some $\delta>0$. This contradicts to $z_{0}=\inf I$. Thus $z_{0} \equiv 0$ and the proof of Lemma 3.4 is completed.

Now we are ready to give an existence result to system (3.25).

Lemma 3.5 For any $\gamma>\frac{5}{3}$, there is a positive function $y=g(z)$ in $C([0,1]) \cap C^{1}((0,1])$ satisfying (3.25).

Proof We can rewrite (3.25) as follows:

$$
\left\{\begin{array}{l}
g^{\prime}(z)=G(g(z), z)=\frac{\frac{\alpha^{\frac{2}{3}}(\gamma-1)(3 \gamma-5) z}{12 \gamma}}{g^{\frac{3 \gamma-1}{3 \gamma-5}}(z)-\frac{3 \gamma+1}{6 \gamma}} \\
g(1)=1 .
\end{array}\right.
$$

We look for a solution to (3.33) such that

$$
g(z) \in C([0,1]) \cap C^{1}((0,1]), \quad\left(\frac{3 \gamma+1}{6 \gamma}\right)^{\frac{3 \gamma-5}{3 \gamma-1}}<g(z) \leq 1, \quad \forall z \in(0,1] .
$$

Set $\mathbb{R}=\{(z, g(z)) \mid 0 \leq 1-z \leq a, 0 \leq 1-g(z) \leq b\}$ for small $a \in(0,1), b \in\left(0,1-\left(\frac{3 \gamma+1}{6 \gamma}\right)^{\frac{3 \gamma-5}{3 \gamma-1}}\right)$. Then we can easily deduce that

$$
|G(g(z), z)| \leq M, \quad \forall(z, g(z)) \in \mathbb{R}
$$

where $M$ is a positive constant only depending on $\gamma, a, b$.

Since $G(g(z), z)$ is continuous in $\mathbb{R}$, by choosing $h=\min \left\{a, \frac{b}{M}\right\}$, one can show that the solution to the initial value problem (3.32) exists in the neighborhood $0 \leq 1-z \leq h$.

Similarly, we can extend this solution from the left of the neighborhood $0 \leq 1-z \leq h$ step by step. Let the maximum interval of the existence of solutions be $(\alpha, 1]$ for some $\alpha \geq 0$; with $g(z) \in C([\alpha, 1]) \cap C^{1}((\alpha, 1])$, we will show that $\alpha \equiv 0$.

If not, then $\alpha \in(0,1)$. By Lemma 3.4, one has $g(\alpha)>\left(\frac{3 \gamma+1}{6 \gamma}\right)^{\frac{3 \gamma-5}{3 \gamma-1}}$ and $(3.33)_{1}$ is well defined in the small neighborhood of $z=\alpha$ for $C^{1}$ function $g(z)$. Thus similar arguments to those above show that the solution of this initial value problem (3.32) in the neighborhood $\mid \alpha-$ 
$z \mid \leq h_{0}$ for small $h_{0}>0$ exists. That is to say, the solution $g(z)$ can be extended to the interval $\left[\alpha-h_{0}, 1\right]$, which contradicts the fact that $(\alpha, 1]$ is the maximum interval of the existence of solutions. Then we have obtained a $C([0,1]) \cap C^{1}((0,1])$ solution $g(z)$ to the system (3.25).

Finally, by virtue of (3.23) and Lemmas 3.4-3.5, we have obtained the global existence of $y=f(z) \in C([0,1]) \cap C^{1}((0,1])$ to $(3.24)$ and the proof of Theorem 2.2 is complete. According to Lemma 3.4, $f(0)>\left(\frac{3 \gamma+1}{6 \gamma}\right)^{\frac{6}{3 \gamma-1}} f(1)$, and $f(z)=\rho(r, t) a^{3}(t)$, we can deduce from (3.21)-(3.22) the following.

Corollary 3.1 The density at the origin of the center has the following estimate:

$$
\left(\frac{3 \gamma+1}{6 \gamma}\right)^{\frac{6}{3 \gamma-1}}\left[\rho_{0}^{\frac{1-3 \gamma}{6}}\left(a_{0}\right)+\frac{3 \gamma-1}{6} t\right]^{\frac{-6}{3 \gamma-1}}<\rho(0, t)=\frac{f(0)}{f(1)}\left[\rho_{0}^{\frac{1-3 \gamma}{6}}\left(a_{0}\right)+\frac{3 \gamma-1}{6} t\right]^{\frac{-6}{3 \gamma-1}}
$$

and then

$$
\rho(0, t) \rightarrow 0, \quad \text { as } t \rightarrow+\infty .
$$

\section{Competing interests}

The authors declare that they have no competing interests.

\section{Authors' contributions}

All authors contributed equally to the writing of this paper. All authors read and approved the final manuscript.

\section{Author details}

${ }^{1}$ School of Mathematics, Northwest University, Xi'an, 710127, China. ${ }^{2}$ School of Mathematics and Information Science, Henan Polytechnic University, Jiaozuo, Henan Province 454000, P.R. China.

\section{Acknowledgements}

The authors would like to thank the anonymous referees for their valuable suggestions which helped to improve the presentation of the paper. This research is partially supported by National Natural Science Foundation of China 11331005, 11201371, and also supported by Research Fund for the Doctoral Program of Higher Education of China 20136101110015.

Received: 5 February 2015 Accepted: 19 May 2015 Published online: 12 June 2015

\section{References}

1. Liu, TP, Xin, ZP, Yang, T: Vacuum states of compressible flow. Discrete Contin. Dyn. Syst. 4, 1-32 (1998)

2. Danchin, R: Global existence in critical spaces for compressible Navier-Stokes equations. Invent. Math. 141, 579-614 (2000)

3. Feireisl, E, Novotoý, A, Petzeltová, H: On the existence of globally defined weak solutions to the Navier-Stokes equations of isentropic compressible fluids. J. Math. Fluid Mech. 3, 358-392 (2001)

4. Hoff, D: Strong convergence to global solutions for multidimensional flows of compressible, viscous fluids with polytropic equations of state and discontinuous initial data. Arch. Ration. Mech. Anal. 132, 1-14 (1995)

5. Jiang, S, Zhang, P: Global spherically symmetric solutions of the compressible isentropic Navier-Stokes equations. Commun. Math. Phys. 215, 559-581 (2001)

6. Kazhikhov, AV, Shelukhin, $\mathrm{V}$ : Unique global solution with respect to time of initial-boundary value problems for one-dimensional equations of a viscous gas. J. Appl. Math. Mech. 41, 273-282 (1977)

7. Lions, PL: Mathematical Topics in Fluid Dynamics 2, Compressible Models. Oxford Science Publication, Oxford (1998)

8. Matsumura, A, Nishida, T: The initial value problem for the equations of motion of viscous and heat-conductive gases. J. Math. Kyoto Univ. 20, 67-104 (1980)

9. Hoff, D, Serre, D: The failure of continuous dependence on initial data for the Navier-Stokes equations of compressible flow. SIAM J. Appl. Math. 51, 887-898 (1991)

10. Xin, ZP: Blow-up of smooth solution to the compressible Navier-Stokes equations with compact density. Commun. Pure Appl. Math. 51, 229-240 (1998)

11. Gerbeau, JF, Perthame, B: Derivation of viscous Saint-Venant system for laminar shallow water, numerical validation. Discrete Contin. Dyn. Syst., Ser. B B1, 89-102 (2001)

12. Marche, F: Derivation of a new two-dimensional viscous shallow water model with varying topography, bottom friction and capillary effects. Eur. J. Mech. B, Fluids 26, 49-63 (2007)

13. Vaigant, VA, Kazhikhov, AV: On the existence of global solutions of two-dimensional Navier-Stokes equations of a compressible viscous fluid. Sib. Mat. Zh. 36(6), 1283-1316 (1995) (in Russian). Translation in Siberian Math. J. 36(6), 1108-1141 (1995) 
14. Jiang, S: Global smooth solutions of the equations of a viscous, heat-conducting one-dimensional gas with density-dependent viscosity. Math. Nachr. 190, 169-183 (1998)

15. Jiang, S, Xin, ZP, Zhang, P: Global weak solutions to 1D compressible isentropic Navier-Stokes with density-dependent viscosity. Methods Appl. Anal. 12, 239-252 (2005)

16. Jiu, QS, Wang, Y, Xin, ZP: Stability of rarefaction waves to the 1D compressible Navier-Stokes equations with density-dependent viscosity. Commun. Partial Differ. Equ. 36, 602-634 (2011)

17. Jiu, QS, Xin, ZP: The Cauchy problem for $1 \mathrm{D}$ compressible flows with density-dependent viscosity coefficients. Kinet. Relat. Models 1, 313-330 (2008)

18. Dou, CS, Jiu, QS: A remark on free boundary problem of 1-D compressible Navier-Stokes equations with density-dependent viscosity. Math. Methods Appl. Sci. 33, 103-116 (2010)

19. Li, HL, Li, J, Xin, ZP: Vanishing of vacuum states and blow-up phenomena of the compressible Navier-Stokes equations. Commun. Math. Phys. 281, 401-444 (2008)

20. Mellet, A, Vasseur, A: Existence and uniqueness of global strong solutions for one-dimensional compressible Navier-Stokes equations. SIAM J. Math. Anal. 39, 1344-1365 (2008)

21. Okada, M, Matusu-Necasova, S, Makino, T: Free boundary problem for the equation of one-dimensional motion of compressible gas with density-dependent viscosity. Ann. Univ. Ferrara, Sez. 7: Sci. Mat. 48, 1-20 (2002)

22. Vong, SW, Yang, T, Zhu, CJ: Compressible Navier-Stokes equations with degenerate viscosity coefficient and vacuum II. J. Differ. Equ. 26(5-6), 965-981 (2001)

23. Yang, T, Zhu, CJ: Compressible Navier-Stokes equations with degenerate viscosity coefficient and vacuum. Commun. Math. Phys. 230, 329-363 (2002)

24. Yang, T, Yao, ZA, Zhu, CJ: Compressible Navier-Stokes equations with density-dependent viscosity and vacuum. Commun. Partial Differ. Equ. 26, 965-981 (2001)

25. Perepelitsa, M: On the global existence of weak solutions for the Navier-Stokes equations of a compressible fluid flows. SIAM J. Math. Anal. 38, 1126-1153 (2006)

26. Jiu, QS, Wang, Y, Xin, ZP: Global well-posedness of 2D compressible Navier-Stokes equations with large data and vacuum. arXiv:1202.1382

27. Huang, XD, Li, J: Existence and blowup behavior of global strong solutions to the two-dimensional barotropic compressible Navier-Stokes system with vacuum and large initial data. Preprint. arXiv:1205.5342

28. Jiu, QS, Wang, Y, Xin, ZP: Global well-posedness of the Cauchy problem of 2D compressible Navier-Stokes equations in weighted spaces. arXiv:1207.5874

29. Huang, XD, Li, J: Global well-posedness of classical solutions to the Cauchy problem of two-dimensional barotropic compressible Navier-Stokes system with vacuum and large initial data. Preprint. arXiv:1207.3746

30. Jiu, QS, Wang, Y, Xin, ZP: Global classical solutions to the two-dimensional compressible Navier-Stokes equations in $\mathbb{R}^{2}$. arXiv:1209.0157

31. Guo, ZH, Jiu, QS, Xin, ZP: Spherically symmetric isentropic compressible flows with density-dependent viscosity coefficients. SIAM J. Math. Anal. 39, 1402-1427 (2008)

32. Guo, ZH, Li, HL, Xin, ZP: Lagrange structure and dynamical for spherically symmetric compressible Navier-Stokes equations. Commun. Math. Phys. 309, 371-412 (2012)

33. Chen, $\mathrm{P}, \mathrm{Zhang}, \mathrm{T}$ : A vacuum problem for multidimensional compressible Navier-Stokes equations with degenerate viscosity coefficients. Commun. Pure Appl. Anal. 7, 987-1016 (2008)

34. Deng, YB, Xiang, JL, Yang, T: Blowup phenomena of solutions to Euler-Poisson equations. J. Math. Anal. Appl. 286, 295-306 (2003)

35. Yuen, MW: Analytical solutions to the Navier-Stokes equations. J. Math. Phys. 49, 102-113 (2008)

36. Yeung, LH, Yuen, MW: Analytical solutions to the Navier-Stokes equations with density-dependent viscosity and with pressure. J. Math. Phys. 50, 083 (2009)

37. Guo, ZH, Xin, ZP: Analytical solutions to the compressible Navier-Stokes equations with density-dependent viscosity coefficients and free boundaries. J. Differ. Equ. 252, 1-19 (2012)

\section{Submit your manuscript to a SpringerOpen ${ }^{\circ}$ journal and benefit from:}

- Convenient online submission

Rigorous peer review

- Immediate publication on acceptance

- Open access: articles freely available online

- High visibility within the field

- Retaining the copyright to your article 